POZZATTI, P.N. et al. Análise hematológica e bioquímica da bioatividade da erva-de-santamaria em bovinos. PUBVET, Londrina, V. 5, N. 39, Ed. 186, Art. 1255, 2011.

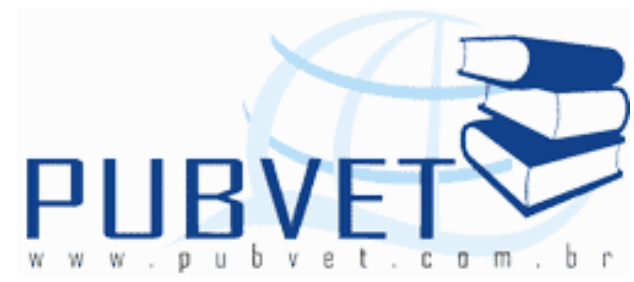

PUBVET, Publicações em Medicina Veterinária e Zootecnia.

\title{
Análise hematológica e bioquímica da bioatividade da erva-de-santa- maria em bovinos
}

\footnotetext{
${ }^{1}$ Profissional Médico Véterinário formado pela Universidade Federal do Espírito Santo.

${ }^{2}$ Professora Doutora do Departamento de Medicina Veterinária do Centro de Ciências Agrárias da Universidade Federal do Espírito Santo.

${ }^{3}$ Doutorando do Departamento de Clínica e Cirurgia da Escola de Veterinária da Universidade Federal de Minas Gerais.

${ }^{4}$ Granduando do curso de Medicina Veterinária da Universidade Federal do Espírito Santo.
}

\section{Resumo}

A erva-de-santa-maria (Chenopodium ambrosioides L.) é uma das plantas usadas na fitoterapia humana, tendo sua origem na América tropical e subtropical, Antilhas e México, sendo muito abundante no Brasil. $O$ Rhiphicephalus (Boophilus) microplus é conhecido no Brasil como carrapato dos bovinos, sendo parasita de alta importância, produzindo perdas diretas e indiretas pela transmissão de doenças e alto custo de seu combate. Este trabalho avaliou os resultados da erva-de-santa-maria sobre os carrapatos, 
POZZATTI, P.N. et al. Análise hematológica e bioquímica da bioatividade da erva-de-santamaria em bovinos. PUBVET, Londrina, V. 5, N. 39, Ed. 186, Art. 1255, 2011.

para controle do R. (Boophilus) microplus através do teste in vivo. Esse teste foi realizado com 10 bovinos adultos, machos sendo banhados via pour on com $30 \mathrm{~mL}$ de loção a $0,2 \%$ de óleo essencial. O produto mostrou repelência por um período de 28 dias. Para verificação das funções renal e hepática, foram realizados testes hematológicos e bioquímicos. Os exames hematológicos não apresentaram alterações significativas enquanto que os bioquímicos mostraram alterações nos analitos creatinina, alanina amino transferase (ALT), aspartato amino transferase (AST) e albumina.

Palavras-chave: teste in vivo, Chenopodium ambrosioides, mastruz.

\title{
Hematological and biochemical analysis of the bioactivity of the wormseed in cattle
}

\begin{abstract}
The wormseed (Chenopodium ambrosioides L.) is one the plants used in herbal medicine human, having its origin in tropical and subtropical America, Caribbean and Mexico, being very abundant in Brazil. The Rhiphicephalus (Boophilus) microplus known in Brazil as the cattle tick parasite being of high importance, producing direct and indirect losses for the transmission of diseases and high cost of combating it. This study evaluated the results of the wormseed for control of $R$. (Boophilus) microplus by testing in vivo. This test was conducted with 10 adult male bovine animals was given a bath to pour on $30 \mathrm{~mL}$ of $0,2 \%$ lotion essential oil. The product showed repellency for a period of 28 days. For verification of the kidney and liver function tests were performed hematological na biochemical parameters. The blood tests showed no significant changes while the biochemical analytes showed changes in creatinine, alanine aminotransferase (ALT), aspatate aminotransferase (AST) and albumin.
\end{abstract}

Keywords: In vivo test, Chenopodium ambrosioides, wormseed 
POZZATTI, P.N. et al. Análise hematológica e bioquímica da bioatividade da erva-de-santamaria em bovinos. PUBVET, Londrina, V. 5, N. 39, Ed. 186, Art. 1255, 2011.

\section{INTRODUÇÃO}

A fitoterapia é o ramo da ciência médica que utiliza plantas, drogas vegetais e seus preparados para o tratamento de enfermidades (SARTI e CARVALHO, 2004). A medicina verde, como também é conhecida, é tão antiga quanto o gênero humano. Pré-hominídeos, pitencantropos, sinantropos ou africantropos guiados pelo mesmo instinto que os gatos e os cachorros quando comem ervas para purgar-se, sabiam distinguir as plantas comestíveis daquelas que podiam curar, cicatrizar ou aliviar (RUDDER, 2002). Em meados do século XIX, as plantas medicinais perderam sua importância e passaram a ser utilizadas como terapia alternativa no Brasil (SARTI e CARVALHO, 2004). Roel (2001) aponta o uso de plantas medicinais como fator importante a ser considerado nos programas de pesquisa em desenvolvimento local e na busca da sustentabilidade na agricultura, principalmente quando se trata de pequenas propriedades em regiões economicamente desfavorecidas.

A erva-de-santa-maria (Chenopodium ambrosioides L.) é uma das plantas usadas na fitoterapia humana, tendo sua origem na América tropical e subtropical, Antilhas e México. Muito abundante no Brasil, possui efeito antihelmíntico pela ação do princípio ativo ascaridol, encontrado em maiores concentrações no óleo essencial, e atua paralisando a musculatura do parasito (CAMARGO, 1985). Possuem ainda como princípios ativos os glicosídios cianogenéticos, saponina (PROJETO GUANDU, 1995), cineol, cimeno, salicilato de metila, quenopodina, histamina, limaneno, glicol, ácidos butírico e salicílico (ALBUQUERQUE, 1989). Estudos indicam ação inseticida (DELOBEL e MALONGA, 1987), repelente (MAZZONETTO, 2002), ação antifúngica (JARDIM, 2008) e o uso tópico das folhas são eficazes no tratamento de Leishmaniose cutânea (PATRÍciO et al. 2008).

Os carrapatos e as doenças por eles transmitidas são amplamente distribuídos pelo mundo, particularmente em regiões tropicais e subtropicais. As infestações bovinas por carrapatos Rhipicephalus (Boophilus) microplus 
POZZATTI, P.N. et al. Análise hematológica e bioquímica da bioatividade da erva-de-santamaria em bovinos. PUBVET, Londrina, V. 5, N. 39, Ed. 186, Art. 1255, 2011.

podem reduzir a produção animal e levar a morte. Este ectoparasita exige um único hospedeiro para a sua evolução e possui sítios de predileção desde a região ventral do pescoço até a genitália do animal. Os danos causados ao hospedeiro são resultantes da ação direta do parasito em consequência da espoliação sanguínea (NETO e TOLEDO, 2006).

O R. (Boophilus) microplus é conhecido no Brasil como carrapato dos bovinos, sendo um parasita de alta importância, produzindo perdas diretas e indiretas pela transmissão de doenças e alto custo de seu combate. Ainda hoje apresenta alta incidência e prevalência no Brasil e em países desenvolvidos de clima tropical, como a Austrália. Provavelmente isso ocorra pela complexidade dos fatores envolvidos no seu combate e desconhecimento pelos produtores de informações específicas e necessárias à adoção de práticas efetivas de controle em suas propriedades (ROCHA, 2005).

O controle dos carrapatos deve ser feito através de práticas de manejo e produtos químicos que acarretam custos elevados, ocorrência de resíduos na carne e no leite, além do rápido desenvolvimento de resistência pelos carrapatos aos princípios ativos utilizados (FRAGA et al. 2003). Essa resistência é de origem genética dependendo da espécie envolvida e pode ser caracterizada por modificações no ciclo biológico (NOLAN, 1981).

Diante das informações apresentadas sobre o R. (Boophilus) microplus visando o controle desse ectoparasito e minimização do custo de tratamento, torna-se necessário o estudo sobre a utilização do Chenopodium ambrosioides L. sobre o controle desse artrópode diretamente sobre os bovinos, para sua utilização no cotidiano da propriedade rural. Isso diminui o número de aplicações dos carrapaticidas químicos, reduzindo seus efeitos, os custos de produção, o grau de espoliação dos animais e a população de $R$. (Boophilus) microplus nas pastagens durante a fase não parasitária, além de reduzir o impacto ambiental causada pelo uso de produtos químicos. 
POZZATTI, P.N. et al. Análise hematológica e bioquímica da bioatividade da erva-de-santamaria em bovinos. PUBVET, Londrina, V. 5, N. 39, Ed. 186, Art. 1255, 2011.

\section{MATERIAIS E MÉTODOS}

Foram selecionados 10 bovinos adultos, machos e criados a pasto da Fazenda Experimental da Universidade Federal do Espírito Santo com sede em São José dos Calçados, região sul do Espírito Santo, sem uso de carrapaticida. Os animais receberam uma única aplicação na linha dorsal (via pour on) de 30 $\mathrm{mL}$ da loção de óleo essencial, extraído por hidrodestilação com aparelho de Clevenger, de erva-de-santa-maria a 0,2\%. A metodologia utilizada para o banho dos animais é a aplicada a bovinos de Martins e González (2007) que utilizaram Citronela de java. As observações individuais ocorreram antes (M0) e após 14 dias (M1) da administração do produto, com acompanhamento visual e verificação da presença de teleóginas sobre os animais aos 7, 14, 21 e 28 dias, bem como avaliação clínica e laboratorial antes e 14 dias após aplicação e análise estatística dos hemogramas e exames bioquímicos realizados.

$\mathrm{Na}$ avaliação clínica, foi verificada as seguintes variáveis como a observação quanto à presença ou não de ectoparasitos e o nível de desidratação dos animais selecionados.

Para a avaliação laboratorial tanto hematológica quanto bioquímica, as coletas foram realizadas com agulhas de calibre $40 \times 12$ de diâmetro, através da veia jugular e armazenada em frascos com anticoagulante EDTA para o hemograma e em frascos sem anticoagulante para as dosagens bioquímicas. As análises foram realizadas no Laboratório de Análises Clínicas do Hospital Veterinário da Universidade Federal do Espírito Santo, antes e após 14 dias do tratamento. O hemograma foi executado por técnicas manuais estabelecidas pelo laboratório para os seguintes parâmetros: hematócrito $(\mathrm{Ht})$, pela técnica do microhematócrito; hemoglobina $(\mathrm{Hb})$ por espectrofotometria; hematimetria (Hm) e leucometria (Lt) pela câmara de Neubauer, seguindo técnicas preconizadas por Jain (1993). As análises bioquímicas séricas foram determinadas a temperatura de $37^{\circ} \mathrm{C}$, com reagentes comerciais Labtest ${ }^{\circledR}$ em 
POZZATTI, P.N. et al. Análise hematológica e bioquímica da bioatividade da erva-de-santamaria em bovinos. PUBVET, Londrina, V. 5, N. 39, Ed. 186, Art. 1255, 2011.

espectrofotômetro SP-22 Bioespectro. A atividade da AST foi determinada pelo método ultravioleta (UV) otimizado, sem piridoxal fosfato. Foram determinadas também proteína plasmática total (PT), albumina (Alb), uréia (Ur), creatinina (Crea) e aspartato amino transferase (AST) e alanina amino transferase (ALT)

Os dados da avaliação laboratorial foram submetidos ao teste $T$ paramétrico para analisar a diferença estatística entre duas amostras dependentes e do mesmo tamanho, onde cada indivíduo é o seu próprio controle, antes e depois. No programa BioEstat 5.0 obteve-se valores médios e respectivos desvio padrão para cada variável comparados com os valores de referência hematológica para a espécie bovina segundo Feldman, Zinkl e Jain (2000), e para os exames bioquímicos conforme Kaneko, Harvey e Bruss (1997).

\section{RESULTADOS E DISCUSSÕES}

Foram avaliados os parâmetros hematológicos conforme ilustram as tabelas 1 e 2 respectivamente. Já as avaliações dos parâmetros bioquímicos estão listadas na tabela 3. Esses resultados são comparados aos valores de referências nas tabelas presentes em anexo.

Avaliaram-se os constituintes do eritrograma dos animais conforme Weiss e Perman (1992), no momento zero (M0) antes do tratamento e no momento um (M1), 14 dias após o tratamento. Os resultados do eritrograma mostraram todos os parâmetros dentro da normalidade, quando comparado entre si e com os valores de referência. 
POZZATTI, P.N. et al. Análise hematológica e bioquímica da bioatividade da erva-de-santamaria em bovinos. PUBVET, Londrina, V. 5, N. 39, Ed. 186, Art. 1255, 2011.

Tabela 1. Avaliação de Hemácias (Hm), Hemoglobina (Hb), Hematócrito (Ht), Volume Globular Médio (VGM) e Concentração de Hemoglobina Globular Média (CHGM) dos animais testados via Pour on a uma concentração de $0,2 \%$ de óleo essencial de erva de santa maria como loção no momento inicial (M0) e final (M1) do experimento

\begin{tabular}{ccccccccccc}
\hline Animal & HmMO & HmM1 & HbMO & HbM1 & HtMo & HtM1 & VGMMO & VGMM1 & CHGMMO & CHGMM1 \\
\hline 1 & 8,15 & 7,45 & 9,5 & 9,6 & 29 & 33 & 35 & 44 & 32 & 29 \\
2 & 7,5 & 6,35 & 9,7 & 8,8 & 30 & 30 & 40 & 47 & 32 & 29 \\
3 & 9,1 & 8,8 & 14,4 & 12,8 & 45 & 42 & 49 & 47 & 32 & 30 \\
4 & 7,8 & 6,8 & 10,2 & 9,5 & 32 & 31 & 41 & 45 & 31 & 30 \\
5 & 7,2 & 6,4 & 12 & 10,5 & 35 & 33,5 & 48 & 52 & 34 & 31 \\
6 & 7 & 7,85 & 11 & 12 & 35 & 39 & 50 & 49 & 31 & 30 \\
7 & 8,45 & 9,5 & 11,7 & 12,4 & 39 & 42 & 46 & 44 & 30 & 29 \\
8 & 7,7 & 7,4 & 12,3 & 11 & 36 & 36 & 46 & 48 & 34 & 30 \\
9 & 7,6 & 9,1 & 11 & 11,3 & 34 & 43 & 44 & 47 & 32 & 26 \\
10 & 8,2 & 8,5 & 10,5 & 10,3 & 34 & 35 & 41 & 41 & 30 & 29 \\
\hline
\end{tabular}

Tabela 2. Leucócitos (Le), Bastões (Ba), Segmentados (Se), Linfócitos (Li), Eosinófilos (Eo) e Monócitos (Mo) dos animais testados via "Pour on" a uma concentração de $0,2 \%$ de óleo essencial de erva de santa maria como loção no momento zero (M0) e um (M1) do experimento.

\begin{tabular}{cccccccccccccc}
\hline Animal & Le MO & Le M1 & BaMO & BaM1 & SeMO & SeM1 & Li MO & LiM1 & EoM0 & EoM1 & MoM0 & MoM1 \\
\hline 1 & 7,800 & 9,900 & 0 & 99 & 1,078 & 1,584 & 5,390 & 6,930 & 616 & 990 & 616 & 297 \\
2 & 8,000 & 7,600 & 0 & 76 & 2,800 & 1,976 & 3,600 & 4,636 & 1,200 & 760 & 400 & 228 \\
3 & 16,100 & 14,200 & 0 & $142 p$ & 2,254 & 2,840 & 11,270 & 9,372 & 1,449 & 1,562 & 1,127 & 284 \\
4 & 10,400 & 7,500 & 0 & 0 & 728 & 975 & 7,280 & 5,700 & 208 & 375 & 2,184 & 450 \\
5 & 7,900 & 8,700 & 0 & 0 & 1,264 & 1,392 & 5,372 & 5,829 & 948 & 1,131 & 316 & 348 \\
6 & 12,000 & 13,700 & 0 & 0 & 2,880 & 4,658 & 6,000 & 7,124 & 1,800 & 959 & 1,320 & 959 \\
7 & 8,400 & 10,100 & 0 & 101 & 2,520 & 2,525 & 4,956 & 6,363 & 504 & 505 & 420 & 606 \\
8 & 11,300 & 11,000 & 0 & 0 & 2,034 & 1,430 & 7,006 & 8,690 & 1,130 & 220 & 1,130 & 660 \\
9 & 11,200 & 13,100 & 0 & 0 & 1,120 & 3,013 & 7,840 & 9,170 & 1,232 & 262 & 1,008 & 655 \\
10 & 11,800 & 13,600 & 0 & $136 p$ & 1,534 & 3,808 & 7,670 & 7,752 & 1,770 & 1,088 & 826 & 816 \\
\hline
\end{tabular}


POZZATTI, P.N. et al. Análise hematológica e bioquímica da bioatividade da erva-de-santamaria em bovinos. PUBVET, Londrina, V. 5, N. 39, Ed. 186, Art. 1255, 2011.

No leucograma houve diferença significativa $(p<0,01)$ entre o M0 e M1, apresentando os animais 3 e 10 valores fora da normalidade, em relação aos neutrófilos bastonetes indicando resposta a processos inflamatórios, justificado pelas possíveis lesões provocadas pelo estágio larval da mosca Dermatobia hominis, que na avaliação clínica foi observada em grande quantidade nos animais selecionados. Com a aplicação do produto testado, as larvas presentes morreram formando abscessos no tecido subcutâneo, sendo este fato caracterizado pela resposta com aumento dos monócitos indicando reparação tecidual, como relatam Meyer, Coles e Rich (1995) e Garcia-Navarro (2005).

Tabela 3. Dosagens de Uréia (Ur), Creatinina (Cr), Albumina (Alb), Aspartato aminotransferase (AST), Alanina aminotransferase (ALT) e Proteína Total (PT) dos animais testados via Pour on a uma concentração de $0,2 \%$ de óleo essencial de erva de santa maria como loção no momento inicial (M0) e final (M1) do experimento

\begin{tabular}{ccccccccccccc}
\hline Teste & UrM0 & UrM1 & CrM0 & CrM1p & AlbMIOp & AlbM1p & ASTMOp & ASTM1p & ALTM0p & ALTMf1p & PTM0 & PTM1 \\
\hline 1 & 36 & 36 & 1,6 & 1,7 & 2.3 & 2.5 & 57 & 108 & 28 & 38 & 8.7 & 8.2 \\
2 & 26 & 30 & 1,9 & 2,2 & 2.7 & 2.8 & 33 & 87 & 22 & 28 & 9.3 & 9.2 \\
3 & 30 & 34 & 1,7 & 1,8 & 2.3 & 2.6 & 46 & 100 & 26 & 26 & 8.7 & 9.4 \\
4 & 29 & 30 & 1,7 & 2,1 & 2.4 & 2.7 & 23 & 88 & 13 & 23 & 7.0 & 8.2 \\
5 & 29 & 30 & 1,3 & 1,8 & 2.5 & 2.7 & 33 & 80 & 15 & 18 & 9.4 & 9.4 \\
6 & 26 & 26 & 1,4 & 1,5 & 2.7 & 2.9 & 33 & 80 & 22 & 22 & 8.2 & 8.7 \\
7 & 34 & 27 & 1,7 & 1,7 & 2.8 & 3.0 & 58 & 73 & 31 & 28 & 8.6 & 9.0 \\
8 & 25 & 25 & 1,6 & 1,9 & 2.4 & 2.5 & 58 & 87 & 16 & 21 & 9.1 & 9.3 \\
9 & 31 & 36 & 1,7 & 1,9 & 2.3 & 3.2 & 46 & 100 & 18 & 22 & 8.0 & 8.2 \\
10 & 22 & 23 & 1,6 & 1,5 & 2.6 & 2.9 & 23 & 57 & 22 & 22 & 9.1 & 9.4 \\
\hline
\end{tabular}

$\mathrm{p}=$ alterações significativas pelo método estatístico utilizado

Nos testes bioquímicos, a avaliação da aspartato aminotransferase (AST) foi em conformidade com Meyer e Harvey (1998); Ortolani et al., (2002); Kerr (2003) e Jackson (2007) e foi constatada diferença significativa $(p<0,01)$ 
POZZATTI, P.N. et al. Análise hematológica e bioquímica da bioatividade da erva-de-santamaria em bovinos. PUBVET, Londrina, V. 5, N. 39, Ed. 186, Art. 1255, 2011.

entre M0 de $41 \mathrm{U} / \mathrm{L} \pm 13,8$ e M1 de $86 \mathrm{U} / \mathrm{L} \pm 14,7$. Para Kaneko, Harvey e Bruss (1997), esses valores apresentam-se aumentados no M1, mas para Gregory et al. (1999) os valores de referência nacionais para a AST são iguais

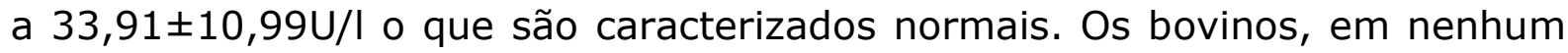
momento, apresentaram sinais clínicos sugestivos de doença hepática comparando os dois momentos quanto ao exame clínico, mas essa diferença deve ser investigada, principalmente com as quantificações de enzimas mais específicas do músculo como a creatinoquinase conforme Jackson (2007).

Para a alanina aminotransferase (ALT) apesar de haver diferença entre os dois momentos do tratamento dos bovinos com erva-de-santa-maria, os valores da enzima ALT se mantiveram dentro da normalidade, quando comparados com Jackson (2007) e Kaneko, Harvey e Bruss (1997), que afirmaram ainda que a ALT não possui valor diagnóstico para alterações hepáticas em bovinos.

A albumina, neste trabalho, apresentou aumento significativo $(p<0,01)$ com 2,5 mg/L $\pm 0,18$ (M0) e 2,7 mg/dL $\pm 0,22$ (M1). No período de avaliação, os animais apresentaram sinais de desidratação ao exame clínico, corroborando Ortoloni et al. (2002); Jackson (2007); Kaneko, Harvey e Bruss (1997); Russell e Roussell (2007) e discordando dos achados de Pereira et al. (2010), que afirmaram que quando ocorre o tratamento subcrônico com doses mais altas de Chenopodium ambrosioides, há redução da concentração de albumina no grupo testado.

Para a creatinina, obteve-se média e desvio padrão no M0 1,62 mg/dL \pm 0,16 e M1 1,81 $\pm 0,22$ com diferença, sendo que $20 \%$ dos animais apresentaram aumento de creatinina, devendo ser melhor investigado este aumento significativo antes e após o tratamento. Como já dito, os animais apresentavam sinais de desidratação e não foi realizada urinálise dos mesmos para confirmação de danos renais. A desidratação associada com o aumento de creatinina podem condizer com os achados de Ortolani et al. (2002) e Russell e Roussell (2007). 
POZZATTI, P.N. et al. Análise hematológica e bioquímica da bioatividade da erva-de-santamaria em bovinos. PUBVET, Londrina, V. 5, N. 39, Ed. 186, Art. 1255, 2011.

Os animais selecionados aleatoriamente para a realização deste experimento pertenciam a um rebanho com escassez de alimentos e suplementos possuindo consequentemente, grandes déficits nutricionais, além da desidratação presente ao exame clínico dos mesmos. Com essas condições supracitadas, torna-se inviável afirmar que as alterações hematológicas e bioquímicas encontradas no presente trabalho são conseqüências exclusivamente dos efeitos do $C$. ambrosioides, havendo a necessidade de novos estudos para a confirmação dos achados laboratoriais.

\section{CONCLUSÕES}

O uso de 0,2\% do óleo de erva-de-santa-maria diluído em álcool, na formulação de loção e aplicação na forma de "pour on" foi eficaz no controle e repelência de R.(Boophilus) microplus por 28 dias.

Os exames hematológicos e bioquímicos apresentaram poucas alterações, considerando a presença de desidratação, sugerindo novas análises para que essas sejam atribuídas ao uso de erva de santa maria.

\section{REFERÊNCIAS BIBLIOGRÁFICAS}

AlBuQUerQUe, J.M. Plantas medicinais de uso popular. Brasília: ABEAS; 1989. 100 p.

CAMARGO, M.T.L.A. Medicina popular. São Paulo: Almed; 1985,130 p.

DELOBEL, A.; MALONGA, P. Insecticidal properties of six plant materials against Caryedon serratus (Olivier) (Coleoptera: Bruchidae). Journal of Stored Products Research. v 23, n. 3, p. 173-176. 1987.

FELDMAN, B.F.; ZINKL, J.G.; JAIN, N.C. Schalm's Veterinary Hematology. 5. ed., Philadelphia: Lippincott Williams \& Wilkins, 2000. 1076p.

FRAGA, A.B. et al. Análise de fatores genéticos e ambientais que afetam a infestação de fêmeas bovinas da raça Caracu por carrapatos (Boophilus microplus). Revista Brasileira de Zootecnia. v. 32, n. 6, p.1578-1586, 2003.

GARCIA-NAVARRO, C.E.K. Manual de Hematologia Veterinária. 2. ed.São Paulo: Varela, 2005. 
GREGORY, L. et al. Valores de referência da atividade enzimática da aspartatoaminotransferase e da gama glutamiltransferase em bovinos da raça Jersey. Influência dos fatores etários, sexuais e da infecção pelo vírus da leucose dos bovinos. Arquivo Brasileiro de Medicina Veterinária e Zootecnia. v.51, n.6, p. 515-22,1999.

JACKSON, M.L. Veterinary clínical pathology. An introduction. Australia: Bçackwel. 2007, $262 p$.

JAIN, N.C. Essentials Veterinary Hematology, Filadélfia: Léa \& Fediger. 1993.

JARDIM, C. M. et al. Composition and Antifungal Activity of the Essential Oil of the Brazilian Chenopodium ambrosioides L. Journal of Chemical Ecology, v.34, n.9, p. 1213-1218, set, 2008.

KANEKO, J.J.; HARVEY, J.W.; BRUSS, M.L. Clinical Biochemistry of Domestic Animal. 5. ed., London: Editora Academic Press, 1997. p.890-894.

KERR, M.G. Exames Laboratoriais em Medicina Veterinária. Bioquímica Clínica e Hematologia. 2 ed. São Paulo: Roca. 2003.

MARTINS, R.M.; GONZÁLEZ, F.H.D. Uso del aceite de citronela de Java (Cymbopogon winterianus Jowitt) (Panicoidideae) como acaricida frente a la garrapata Boophilus microplus Canestrini (Acari:Ixodidae). Revista Brasileira de Plantas Medicinais, v.9, n.4, p.1-8, 2007.

MAZZONETTO, F. Efeito de genótipos de feijoeiro e de pós de origem vegetal sobre Zabrotes subfasciatus (Boh.) e Acanthoscelides obtectus (Say) (Col.: Bruchidae). 2002. 134p. (Tese de doutorado), Programa de Pós Graduação da Escola Superior de Agricultura "Luiz de Queiroz", USP, Piracicaba, São Paulo, 2002.

MEYER, D.J.; COLES, E.H.; RICH, L.J. Medicina de laboratório veterinária: interpretação e diagnóstico. São Paulo: Editora Roca, 1995. p.83-90.

MEYER, D.J; HARVEY, J.W. Veterinary Laboratory Medicine. Interpretation e Diagnosis. 2. ed. London: W.B. Sauders Company. 1998. 373p.

NETO, S.F.P.; TOLEDO, P.E.A. Análise da eficiência de carrapaticidas contra Boophilus microplus em gado leiteiro. Revista Científica Eletrônica De Medicina Veterinária. v.3, n. 7, jun 2006. Disponível em: http:// www.revista. inf.br/ veterinária 07/ artigos/edic08artgo07.pdf. Acesso em: 15 mai 2009.

NOLAN, J. Current developments in resistance to adimine and pyrethroids tickicides in Australia. In: Internatinal Conference held from 27. Rhodes University. jan 1981.

ORTOLANI, N. P. et al. Avaliação metabólico-nutricional de vacas leiteiras por meio de fluídos corporais, sangue, leite e urina. Anais do Curso no $29^{\circ}$ Congresso Nacional de Medicina Veterinária. Gramado, RS, 2002.

PATRÍCIO, F.J. et al. Efficacy of the intralesional treatment with Chenopodium ambrosioides in the murine infection by Leishmania amazonensis. Journal of Ethnopharmacology. v.115, n.2, p.313-319. 2008.

PEREIRA, W.S. et al. Evaluation of the subchronic toxicity of oral treatment with Chenopodium ambrosioides in mice. Journal of Ethnopharmacology. v. 127, n.3, p. 602-605, 2010.

PROJETO GUANDU. Terra, água e chá. $1^{\text {a }}$ ed. Vitória: ITA; p.311, 1995. 
ROCHA,C.M.B.M. Caracterização da percepção dos produtores de leite do município de Divinópolis/Mg sobre a importância do carrapato Boophilus microplus e fatores determinantes das formas de combate utilizadas. (Dissertação de mestrado). 2005. Programa de pós-graduação da Escola de Veterinária- UFMG, Belo Horizonte. Minas Gerais. p.1996. 2005.

ROEL, A.R. Utilização de plantas com propriedades inseticidas: uma contribuição para o Desenvolvimento Rural Sustentável. Revista Internacional de Desenvolvimento Local, v.1, n.2, p.43-50, 2001.

RUDDER, E.A.M.C. Guia Compacto das Plantas Medicinais. São Paulo: Ed. Ridell; 2002, $478 \mathrm{p}$.

RUSSELL, K.E.; ROUSSEL, A.J. Evaluation of the Ruminant Serum Chemistry Profile. Veterinary Clinics of North America: Food Animal Pratice. v. 23, n.3, p.403-426, 2007.

SARTI, S. J.; CARVALHO, J. C. T. In: CARVALHO, J. C. T. Fitoterápicos e anti-inflamatórios: aspectos químicos, farmacológicos e aplicações terapêuticas. Fitoterapia e fitoterápicos. Ribeirão Preto. São Paulo: Tecmed; p.13-38, 2004.

WEISS, D.J.; PERMAN, V.P. Assessment of the hematopoietic system in ruminants. Veterinary Clinics of North America: Food Animal Pratice. v.8, n.2, p.411-429, 1992. 


\section{ANEXO}

Tabela 1. Valores de referência de hemograma para bovinos adultos.

\begin{tabular}{|c|c|c|c|}
\hline Eritrograma & $\begin{array}{c}\text { Valores } \\
\text { Referências* }\end{array}$ & Leucograma & $\begin{array}{c}\text { Valores } \\
\text { Referências* }\end{array}$ \\
\hline Hematimetria $(\mathrm{Hm})$ & $5,0-10,0 \times 10^{6} \mu \mathrm{L}$ & $\begin{array}{l}\text { Leucócitos } \times 10^{3} / \mathrm{uL} \\
\text { (Le) }\end{array}$ & $4.0-12.0$ \\
\hline Hemoglobinometria ( $\mathrm{Hb})$ & $8-15 \mathrm{~g} / \mathrm{dl}$ & Bastões /uL (Ba) & $0-120$ \\
\hline Hematócrito (Ht) & $24-46 \%$ & $\begin{array}{l}\text { Segmentados /uL } \\
(\mathrm{Se})\end{array}$ & $600-4.000$ \\
\hline $\begin{array}{l}\text { Volume Globular Médio } \\
\text { (VGM) }\end{array}$ & $40-60 \mathrm{fL}$ & Linfócitos /uL (Li) & $2.500-7.500$ \\
\hline $\begin{array}{l}\text { Concentração de } \\
\text { Hemoglobina }\end{array}$ & $30-36 \%$ & Eosinófilos /uL (Eo) & $0-2.400$ \\
\hline Globular Média (CHGM) & & Monócitos /uL (Mo) & $25-840$ \\
\hline
\end{tabular}

*FELDMAN, B.F.; ZINKL, J.G.; JAIN, N.C. Schalm's Veterinary Hematology. 5 a ed., Philadelphia: Lippincott Williams \& Wilkins, 2000. 1076p

Tabela 2. Valores de referência de bioquímica para bovinos

\begin{tabular}{|c|c|c|}
\hline Bioquímica & & $\begin{array}{c}\text { Valores } \\
\text { Referências* }\end{array}$ \\
\hline $\begin{array}{l}\text { Ureia } \\
\text { (Ur) }\end{array}$ & & $20-30 \mathrm{mg} / \mathrm{dl}$ \\
\hline Creatinina & $(\mathrm{Cr})$ & $1-2 \mathrm{mg} / \mathrm{dl}$ \\
\hline $\begin{array}{l}\text { Albumina } \\
\text { (Alb) }\end{array}$ & & $3,0-3,5 \mathrm{~g} / \mathrm{dl}$ \\
\hline $\begin{array}{l}\text { ALT } \\
(T G P)\end{array}$ & & $11-40 \mathrm{U} / \mathrm{L}$ \\
\hline $\begin{array}{l}\text { AST } \\
(T G O)\end{array}$ & & $78-132 \mathrm{U} / \mathrm{L}$ \\
\hline $\begin{array}{l}\text { Proteína } \\
\text { (PT) }\end{array}$ & & $6,7-7,4 \mathrm{~g} / \mathrm{dl}$ \\
\hline
\end{tabular}

*KANEKO, J.J.; HARVEY, J.W.; BRUSS, M.L. Clinical Biochemistry of Domestic Animal. 5a ed., London: Editora Academic Press, 1997. 890-894p 